\title{
Transferrin receptors of Neisseria meningitidis: promising candidates for a broadly cross-protective vaccine
}

\author{
D. A. A. ALA'ALDEEN \\ Division of Microbiology, Department of Clinical Laboratory Sciences, University Hospital, Queen's Medical \\ Centre, Nottingham NG7 $2 \mathrm{UH}$
}

\begin{abstract}
Production of a meningococcal vaccine capable of generating long-lasting immunity in all age groups is still a high priority worldwide. Iron-regulated outer-membrane proteins have attracted considerable attention in recent years and it has become increasingly evident that the meningococcal transferrin-binding proteins, TBP1 and TBP2, have characteristics compatible with a safe and broadly cross-reactive vaccine candidate. Both TBPs are surface-exposed and immunogenic in man and animals, and antibodies to their native structure are bactericidal to homologous and many heterologous strains. These include strains from various serogroups, serotypes and serosubtypes, with no obvious correlation between bactericidal activity and the identity of the strains or the molecular mass of the heterogeneous TBP2 molecule. A meningococcal vaccine based on, or enriched with, undenatured TBPs from one or more strains, in combination with conventional polysaccharide-based vaccines, might increase the spectrum of strains against which protection can be achieved to include serogroup B strains. In this review, the structure-function and immunological properties of TBP1 and TBP2 are discussed.
\end{abstract}

\section{Introduction}

Neisseria meningitidis, an obligate human pathogen, is the most common cause of pyogenic meningitis worldwide, and is the only bacterium capable of generating outbreaks of bacterial meningitis. It also causes overwhelming meningococcal septicaemia that can kill within hours of the appearance of the first symptoms. Production of a cross-protective vaccine capable of generating long-lasting immunity against the disease among infants and older age groups is still a high priority worldwide. An ideal meningococcal vaccine should be safe; offer long-lasting immunity to all age groups; cross-protect against all meningococcal serogroups, serotypes and serosubtypes; be given orally or nasally; and be easily incorporated into the WHO Expanded Program on Immunisation. So far, no such vaccine has been developed.

However, vaccines based on the serogroup antigens, the capsular polysaccharides, are available against sero-

Received 21 July 1995; accepted 9 Aug. 1995. groups A, C, W135 and Y [1]. These vaccines offer good, but relatively short-lived, protection against their respective serogroups. They do not cross-protect against serogroup B meningococci, which are responsible for the majority of cases in Europe and North, Central and Latin America. In England and Wales, for example, $>70 \%$ of meningococcal infections over the past few years were caused by serogroup $B[2,3]$. Another crucial limitation of these vaccines is that they offer little, if any, protection against infection in infants and children under the age of 2 years - i.e., the most vulnerable age groups. Again in England and Wales, nearly $60 \%$ of cases in 1992 occurred in children under the age of 5 years, of which $40 \%$ were in children aged $<1$ year [2]. Attention has therefore turned to subcapsular meningococcal antigens, particularly the outermembrane proteins (OMPs). These include constitutively expressed and environmentally regulated OMPs. Most investigations on bacterial pathogens, including $N$. meningitidis, are with organisms grown in vitro under conditions that do not necessarily reflect those in vivo. This is likely to give, at best, only a partial picture of bacterial characteristics associated with virulence and with immune responses important for protection. 
The extremely low availability of iron in mammalian tissue fluids constitutes one major difference between the usual growth conditions in vitro and those found in vivo. Pathogens which need to multiply in vivo to establish an infection adapt to this iron-restricted environment by expressing new iron-regulated OMPs that are involved in assimilating protein-bound iron, or in acquiring it from liberated haemoglobin or haem [4]. When grown under conditions of iron restriction e.g., in the presence of an iron chelator such as desferrioxamine - meningococci express several proteins that appear to be suppressed (partially or totally) under iron-sufficient growth conditions. Many of these proteins, which vary in terms of their molecular mass and cellular localisation, are believed to be related directly to iron acquisition from the host's iron-binding proteins and other iron sources. These iron-regulated proteins include two transferrin-binding proteins (TBP1 and TBP2) [5, 6], a 105-kDa lactoferrin-binding protein [7], a $37-\mathrm{kDa}$ periplasmic ironbinding protein $(\mathrm{Fbp})[8]$, an $85-\mathrm{kDa}$ haemoglobinhaptoglobin utilisation protein $(\mathrm{Hpu})$ [9], two RTX cytotoxin-related proteins (a 120-kDa FrpA and a 200$\mathrm{kDa} F r p C)[10,11]$, and a $70-\mathrm{kDa}$ protein $(\mathrm{FrpB})$ of uncertain function $[12,13]$.

\section{Meningococci and iron acquisition from human transferrin}

Iron is present in the human body in large quantities and in high concentration in body fluids $(>20 \mu \mathrm{M})$. However, very little if any of it is available readily to the invading pathogen [4]. Intracellular iron inaccessible to the extracellular organism because of its location - is present in the form of ferritin, haemosiderin and haem. In the extracellular compartment, iron is bound tightly to the glycoproteins (transferrin in serum and lymph, and lactoferrin in the mucosal secretions), which are believed to be the most relevant iron sources for extracellular bacterial infection. Lactoferrin functions more to withhold iron than to transport it, and is found primarily in secretions, whereas transferrin fulfills both functions [4]. Transferrin and lactoferrin have $49.8 \%$ homology in their amino-acid sequence, and both are bilobal structures with the capacity to bind two atoms of ferric iron reversibly in association with the binding of an anion, usually a bicarbonate.

In the in-vivo situation, transferrin is only $30 \%$ saturated with iron, and has an association constant of $c .10^{36}$. The amount of free iron in equilibrium with transferrin within the extracellular fluid is estimated at about $10^{-18}$. As a result, the concentration of free ionic iron in the extracellular compartment is much lower than that required for bacterial growth $(0.05-0.5 \mu \mathrm{M})$ [4]. In addition, the hypoferraemic host response to infection reduces the plasma iron concentration further and also inhibits iron absorption from the gastrointestinal tract. Despite this iron limitation in vivo, pathogenic bacteria are still able to invade, survive, grow and cause disease in the host. This indicates that micro-organisms have acquired efficient scavenging mechanisms for assimilation of protein-bound iron. These include the production of siderophores, low mol. wt high-affinity iron-chelators that can chelate iron from the host's iron-binding proteins.

Although pathogenic neisseria are not able to produce siderophores, they are capable of removing iron from transferrin through a saturable receptor-mediated mechanism that is highly specific for human transferrin [14]. Ala'Aldeen et al. have demonstrated that transferrin binds directly to the surface of meningococci grown in vitro and in vivo [15], and that there is a higher degree of transferrin-binding by iron-starved than iron-sufficient organisms in vitro. With organisms derived without laboratory subculture from the cerebrospinal fluid of a patient, it was shown that organisms grown in vivo also bind transferrin to the same extent as iron-starved cells grown in vitro [15]. It is interesting that there were consistent and considerable cell-to-cell variations in transferrin receptor expression amongst organisms grown either in vitro or in vivo, which could be caused by the fact that cells are not synchronous during growth or may result from possible steric hindrance by capsule or various outermembrane proteins.

The number of transferrin-binding sites on the cell structure has been estimated as a few thousand per meningococcal cell when fully expressed [16]. The glycoproteins are not internalised during the transferrin-binding process [15], thereby differing from the mammalian cell transferrin receptor. The binding of iron-loaded transferrin to the meningococcal surface is an energy-independent process, whereas iron uptake from transferrin is energy-dependent.

It is now clear that the transferrin-receptor is formed, partly or wholly, by the transferrin-binding proteins TBP1 and TBP2. The TBPs were linked previously with biologically functional transferrin receptors in live meningococci [15] by means of rabbit antisera containing antibodies against the TBPs — that inhibited the specific binding between transferrin and live meningococci. Theoretically, it is possible that antibodies against TBPs might interfere with the chelation and uptake of iron from transferrin, and thereby inhibit the survival and growth of the organism in vivo. Lissolo et al. [17] demonstrated the ability of such antibodies to inhibit meningococcal growth in vitro when transferrin was used as the only source of iron. It is not clear whether this effect is mediated entirely by the blocking of transferrin-binding, iron internalisation, or both. 


\section{TBP1 and TBP2: structure analysis}

TBP1 is a $c$. $98-\mathrm{kDa}$ protein that varies in mol. wt only marginally $( \pm c .5 \mathrm{kDa}$ ) between meningococcal strains $[6,18]$. This protein loses its biological activity when exposed to denaturing conditions, such as those used for SDS-PAGE, hence it is not visualised on Western blots. TBP2 (c. $65-90 \mathrm{kDa}$ ) shows considerable molecular heterogeneity amongst different strains of $N$. meningitidis $[6,19]$, and retains its transferrin-binding activity following SDS-PAGE.

Although these two proteins appeared to be coregulated, it was not clear whether they were encoded by contiguous genes. Wilton et al. [20] used a novel method - twin $\mathrm{N}$-terminal polymerase chain reaction - to produce evidence that the genes encoding the two TBPs are organised in tandem, and that the gene encoding TBP2 $(t b p B)$ is immediately upstream of that encoding TBP1 $(t b p A)$. This was confirmed when both genes were cloned [21,22] and sequenced (Accession nos. Z15130 and X75166). The two genes, separated by only a small (87-bp) stretch of DNA, seem to be cotranscribed with a single putative promoter located upstream of $t b p B$ [21]. This promoter region also appears to possess a Fur-binding iron-box, indicating that the $t b p$ genes are also regulated negatively by the combined effect of iron and Fur protein.

Computer-predicted structure analysis of the gene sequences suggests that TBP1 is a gated, transmembranal and porin-like channel with homology to TonBdependent receptors of other gram-negative organisms [21]. It appears that the $\beta$-sheets of this porin form loops, which together create three-dimensional (discontinuous, conformational) transferrin-binding domains that not only hold transferrin, but may also chelate its iron and transport it across the membrane. It is interesting that the complex structure predicted by the sequence-based computer model is in agreement with the structure-function studies which show that only the conformationally stabilised (i.e., native) TBP1 retains its biological and immunological activities [6]. The native structure of TBP1 seems to conceal strongly immunogenic and highly conserved epitopes that are not surface accessible, but which are capable of generating cross-reactive antibodies in animals, as detected by Western blots [18]. However, when the native structure of TBP1 is preserved (and stabilised), it generates cross-reactive antibodies that are detectable on native dot immunoblots, but not Western blots [6].

In contrast, TBP2 appears to be a lipoprotein exported and anchored to the outermost layer of the bacterial cell membrane, with no apparent membrane-embedded domains $[21,23]$. This is supported by the fact that it is more immunogenic than TBP1, and that higher levels of antibodies are generated against its linear epitopes during infection with live organisms. It is noteworthy that the fatty acid moiety of TBP2 does not seem to interfere with the biological and immunological activities of the protein [23].

It is not clear whether TBP1 and TBP2 are linked together physically to form the transferrin receptor, but it is clear that they have a well co-ordinated interaction. They are both surface exposed [15, 24], bind both ironfree (apo-) and iron-loaded (holo-) transferrin, and their joint transferrin-binding activity seems to be localised almost entirely in the $C$-lobe of transferrin [25]. More recently, Kabani et al. [26] used recombinant chimeric molecules of human transferrin to localise the region of human transferrin involved in binding of the meningococcal TBPs to amino-acid residues 365-607. Complementary studies by Gorringe et al. [27] characterised the biologically active linear epitope on TBP2 with murine monoclonal antibodies (MAbs) and multiple overlapping synthetic peptides. Antibodies raised against this linear epitope (residues MRLKRRNWYP) cross-reacted between TBP2 molecules of different strains, including those with either high or low mol. wt TBP2 isotypes.

Work with isogenic mutants suggests that both TBP1 and TBP2 are essential for iron acquisition from transferrin [28]. TBP1, which binds transferrin more strongly than TBP2 [28], does not seem to discriminate between apo-transferrin and holo-transferrin, indicating that it binds domains on transferrin which are not affected by the conformational changes induced by iron-binding, whereas TBP2 appears to be more selective and binds iron-loaded transferrin better than iron-free transferrin [29], indicating that it binds those domains on transferrin which are affected by ironbinding. Gorringe et al. [27] have raised murine MAbs capable of inhibiting transferrin-binding on TBP2, but not TBP1, even though these antibodies cross-reacted with the two TBPs. This is an indication that the two TBPs bind separate parts of transferrin, and that it may be physically possible for both TBP1 and TBP2 to simultaneously bind to a single transferrin molecule before iron chelation. It is possible that TBP2, which is more selective and less membrane-embedded than TBP1, does the primary search for holo-transferrin and brings it closer to TBP1. The two together will then bind the transferrin molecule and induce the necessary conformational changes in transferrin until the bound iron is released and internalised by TBP1. The latter process may be facilitated by other proteins, such as the iron-binding protein Fbp [8]. Therefore, TBP1 is likely to be performing three important functions: namely, binding transferrin and bringing it closer to its micro-environment; chelating the iron from transferrin (assisted in this process by TBP2); and traversing the iron down its pore. It interesting that iron chelation from transferrin is mediated in mammalian cells by conformational changes in transferrin induced by a drop in $\mathrm{pH}$. In meningococci, such iron release must occur on the cell surface, and Gorringe et al. [27] have shown that the release of iron from the 
transferrin-TBP1-TB2 complex occurs at much higher $\mathrm{pH}$ value than from transferrin alone or the transferrinmammalian receptor complex.

\section{In-vivo expression and immunogenicity of the TBPs}

So far, no naturally occurring $\mathrm{TBP}^{-}$clinical meningococcal isolate has been reported, indicating that these proteins are probably vital for survival and growth during infection. It is now clear that both TBP1 and TBP2 generate widely heterogeneous immune responses in vivo, depending on the host species, TBP2 isotype, the vaccine preparation, the route of administration, and other less well understood factors. It has been demonstrated that mice and man infected with live organisms, and rabbits vaccinated with natively purified TBPs, respond only to discontinuous epitopes of TBP1 [6]. In contrast, rabbits infected with live organisms, and mice vaccinated with TBPs, generate antibodies recognising both linear and conformational epitopes of TBP1. However, it is not clear how man would respond to purified TBP preparations. The reasons for differences in the immune responses of the different hosts to the same antigens remain unclear; however, the form and route of antigen presentation in a vaccine, or during infection, may play a part, as well as differences between the immune system of the host species. Whatever the reason, these observations have important implications for assessing the vaccine potential of TBPs and other meningococcal antigens.

Considering the stability of TBP2, in terms of its structural and immunological characteristics, it is not surprising that it has attracted more attention than TBP1. The available data suggest that TBP2 possesses strain-specific and cross-reactive epitopes, as determined by Western blots. Mice, when infected with live organisms or vaccinated with natively purified TBPs, generate strain-specific anti-TBP2 antibodies, whereas similarly treated rabbits generate broadly cross-reactive anti-TBP2 antibodies [6]. It is interesting that patients recovering from natural infection possess fully crossreactive anti-TBP2 antibodies [6].

Rokbi et al. [18] raised rabbit anti-TBP antibodies against gel-extracted TBPs obtained from two different strains, representing strains with low $(<70 \mathrm{kDa})$ and high $(>70 \mathrm{kDa})$ mol. wt TBP2 molecules. The presence of mutually exclusive epitopes that divided the TBP2 molecules into two different families (groups, isotypes) was highlighted. The antigenic heterogeneity correlated well with molecular and genetic heterogeneity. The majority of the strains examined expressed a high mol. wt TBP2 that showed cross-reactivity, but failed to cross-react with the low mol. wt TBP2 isotypes. Ferreirós et al. [30] studied the immunoreactivity of TBP2 molecules of different strains with human convalescent sera, and showed reaction patterns that supported this isotype classification.

Griffiths et al. [31] sequenced the first $20 \mathrm{~N}$-terminal amino-acid residues of the TBP2 molecules from three different strains, two of which expressed high mol. wt TBP2 isotypes and one of which expressed a lower mol. wt TBP2. The amino-acid sequences were identical only up to residue 13 . Antibodies raised to a synthesised peptide based on these common residues (linked to Keyhole Limpet haemocyanin) reacted on immunoblots with the TBP2 of all strains examined, including those with lower or higher mol. wt TBP2 isotypes. Subsequently, Legrain et al. [21] fully sequenced the $t b p B$ genes of the two strains described by Rokbi et al. [18] - which represent the two isotype families - and found only $47 \%$ homology between their deduced TBP2 amino-acid sequences. This contrasts with the $76 \%$ homology found between the deduced amino-acid sequence of their respective TBP1 molecules [21]. The TBP1 sequence and antigenic heterogeniety is much less diverse, and TBP1 Nterminal amino acid sequence data on other strains also support this finding [31].

In this context, it is interesting to know that gonococci express TBPs similar to those expressed by meningococci, and that the DNA and amino-acid sequence analysis of TBP2 from one test strain shows greater homology to the meningococcal TBP2 molecule of the higher mol. wt isotype. This suggests that closer evolutionary links exist between gonococci and those meningococcal strains that express the higher mol. wt TBP2 [32]. Furthermore, there are a number of other neisserial and non-neisserial organisms that are known to express TBPs similar to those of meningococci, and their TBP2 molecules have been shown to cross-react with those of meningococci. These include $N$. lactamica, N. polysacchareae and Haemophilus influenzae $[6,13,33,34]$. Actinobacillus pleuropneumoniae also expresses a TBP2-equivalent transferrin-binding protein that shares $35-38 \%$ sequence homology with meningococcal TBP2 [35]. Therefore, it is possible that the TBP-based iron-acquisition mechanisms in all these organisms have a common origin, and that their TBP genes originate from a common ancestral gene [21].

Finally, with regard to possible cross-reaction between meningococcal and human transferrin receptors, it is now increasingly clear that, despite their common function, these receptors have no common structural or immunological properties. Animal sera raised against meningococcal transferrin receptors fail to recognise human lymphocytes [6] or purified human placental transferrin receptors [34]. The fact that man mounts an immune response to the meningococcal transferrin receptors during infection further supports the evidence that the native structures of these receptors are not seen as self-antigens, otherwise either tolerance and inability to generate antibodies against this protein, or the 
development of post-infection autoimmune diseases in most patients would be expected, and this is not the case.

\section{Bactericidal activity of antibodies to the TBPs}

Protection has been correlated with the presence of bactericidal antibodies [36], and various lines of evidence highlight the importance of humoral bactericidal activity in host defence against $N$. meningitidis. Despite their numerous short-comings, bactericidal assays have become established as the best available test to determine the protective ability of specific antisera raised against vaccine candidates. However, it is not certain to what extent the in-vitro experimental conditions reflect events occurring in vivo. The data linking bactericidal antibodies with protection relate primarily to the group $\mathrm{A}$ and $\mathrm{C}$ polysaccharides, but have been extended to include bactericidal antibodies against outer-membrane proteins.

Danve et al. [37] showed clearly that passive immunisation with rabbit polyclonal antisera raised to affinity-purified TBPs protected mice from a 100 LD50 meningococcal challenge with the homologous strain. Mice immunised actively with three $25-\mu \mathrm{g}$ doses of affinity-purified TBPs adsorbed on aluminium hydroxide or in Freund's adjuvant were also protected from challenge with the homologous strain. No protection against challenge with heterologous strains was reported. However, it was shown with in-vitro bactericidal assays that the rabbit anti-TBP antibodies were bactericidal against five of 10 heterologous strains examined, irrespective of the strain identity in terms of serogroup, serotype and serosubtypes. More recently, Lissolo et al. [17] demonstrated that mice and rabbits immunised with three injections of purified TBP2 (separated from the TBP1-TBP2 complex) produced bactericidal antibodies capable of protecting mice from lethal challenge. However, native TBP1 was not purified and, therefore, the role of this polypeptide in generating protective immunity was not defined. It was concluded that near-native TBP1 is required to study its role in protection.

Ala'Aldeen and Borriello [24] examined the ability of murine and rabbit anti-TBP antisera (raised to natively purified TBPs) to kill homologous and heterologous meningococcal strains, but found no obvious correlations between the bactericidal activity of the antisera and the molecular mass or the Western blot profile of TBP2. Animal antisera were able to kill strains that expressed TBP2 molecules of either higher or lower mol. wt isotypes. Conversely, even strains expressing TBP2 molecules of almost identical mass showed some variation in susceptibility to the sera. Also, the sera killed strains that showed no cross-reactivity on Western blots and, conversely, some strongly crossreactive strains were not killed by these sera. These observations indicated that the bactericidal antibodies were not restricted to those generated against linear epitopes. Western blots could be misleading for detecting antibodies to discontinuous epitopes. While most of the generated anti-TBP1 antibodies react with discontinuous epitopes, antibodies to TBP2 react with continuous epitopes, although some might also be directed against conformational epitopes which may renature sufficiently after SDS-PAGE to allow interaction with both antibodies and transferrin. There are also immune responses to discontinuous epitopes, and Maitre-Wilmotte et al. [38] raised several MAbs to TBP2 that reacted with the native protein (detected by ELISA), but failed to react with linear epitopes on Western blots.

Lissolo et al. [17] demonstrated that purified TBP2 (separated from the TBP1-TBP2 complex) could induce bactericidal antibodies. However, bactericidal activity was not detected in rabbit or mouse polyclonal anti-TBP1 antisera raised to purified and separated TBP1. In contrast, when Ala'Aldeen and Borriello [24] examined the rabbit anti-TBP1-TBP2 complex antiserum against mutant strains that lacked either TBP1 or TBP2, they found that both mutants were as susceptible to the antiserum as the parent strain. Unfortunately, the double knock-out mutant, which lacked both TBP1 and TBP2, was totally sensitive to the complement sources and, therefore, could not be examined for the killing effect of the antiserum. The loss of both TBPs apparently affected other properties of the organism, which rendered it sensitive to killing by complement. The only clear difference between the two studies is in the method of TBP1 preparation. Lissolo et al. [17] separated TBP1 from the TBP1-TBP2 complex by means of a series of potentially denaturing conditions, such as ethanol precipitation and guanidine hydrochloride wash [17], whereas Ala'Aldeen and Boriello [24] made no attempt to separate the native (biologically active) TBP1 from the complex before immunising the rabbit.

More recently, Bishop et al. [39] raised murine antiTBP1 MAbs. One of these antibodies, which recognised a conformational epitope on native dot immunoblots but not Western blots, was bactericidal, while a $\mathrm{MAb}$ that reacted with a linear epitope failed to kill the organism.

It is interesting that Lissolo et al. [17] and Gorringe et al. [27] found that antibodies capable of blocking the biologically active sites of TBP1 or TBP2 were nonbactericidal. Previous work suggested, at least in animals, that the biologically active epitopes are immunogenic and generate blocking antibody responses not only after vaccination with purified TBPs, but also after infection with live meningococci $[15,40]$. In man, it is still not clear whether these transferrin-binding sites behave as they do in animals after vaccination or infection. Whatever the bactericidal activity of anti- 
bodies to biologically active epitopes of either TBP, it seems likely that they could have a detrimental influence on iron acquisition by the organism and its growth. Therefore, the TBPs seem to possess a combination of important epitopes that can generate protective antibodies which can kill the organism by complement mediated bactericidal activity or nutritional starvation. It is possible that a mixture of more than one TBP2 isotype - depending on the prevalent meningococcal strains in any one country - with stabilised native TBP1 molecules will be required to enhance and broaden the protective efficacy of any TBP-based vaccines.

Finally, Ala'Aldeen and Borriello [24] showed that animal anti-TBP1-TBP2 complex antibodies were bactericidal for $N$. polysacchareae - a commensal species that shares very little in terms of serogrouping, serotyping and serosubtyping determinants with meningococci - the test strain of which expresses a low mol. wt TBP2 isotype. This observation indicates that TBP-based vaccines may also influence non-meningococcal neisserial carriage or even protect against gonococcal infection. Further studies are required to address this issue. This also raises the theoretical possibility of cross-protecting against other TBPexpressing non-neisserial organisms by using meningococcal TBPs as vaccines.

In conclusion, it is becoming increasingly evident that the TBPs have characteristics compatible with a safe and broadly cross-protective vaccine candidate. It is clear that TBP1 and TBP2 are both surface-exposed and immunogenic in man and animals, and that antibodies to their native structure are bactericidal to homologous and many heterologous strains. These include strains from various serogroups and serotypes, with no obvious correlation between bactericidal activity and the identity of the strains or the molecular mass of the TBP2 molecule. Although the animal antiTBP antibodies failed to kill some meningococcal strains, it is not clear how human anti-TBP antibodies would behave. A meningococcal vaccine based on, or enriched with, undenatured TBPs in combination with conventional polysaccharide-based vaccines might increase the spectrum of strains against which protection can be achieved to include serogroup B strains.

\section{References}

1. Ala'Aldeen DAA, Griffiths E. Vaccines against meningococcal diseases. In: Ala'Aldeen DAA, Hormaeche CE (eds) Molecular and clinical aspects of bacterial vaccine development. Chichester, John Wiley and Sons. 1995: 1-39.

2. Jones DM, Kaczmarski EB. Meningococcal infections in England and Wales: 1992. CDR Rev 1993; 3: R129-131.

3. Jones DM, Kaczmarski EB. Meningococcal infections in England and Wales: 1993. CDR Rev 1994; 4: R97-R100.

4. Bullen JJ, Griffiths E. Iron and infection: molecular, physiological, and clinical aspects. Chichester, England, John Wiley and Sons. 1987.

5. Schryvers AB, Morris LJ. Identification and characterization of the transferrin receptor from Neisseria meningitidis. Mol Microbiol 1988; 2: 281-288.

6. Ala'Aldeen DAA, Stevenson P, Griffiths $\mathrm{E}$ et al. Immune responses in humans and animals to meningococcal transferrinbinding proteins: implications for vaccine design. Infect Immun 1994; 62: 2984-2900.

7. Schryvers $A B$, Morris LJ. Identification and characterization of the human lactoferrin-binding protein from Neisseria meningitidis. Infect Immun 1988; 56: 1144-1149.

8. Chen CY, Berish SA, Morse SA, Mietzner TA. The ferric ironbinding protein of pathogenic Neisseria spp. functions as a periplasmic transport protein in iron acquisition from human transferrin. Mol Microbiol 1993; 10: 311-318.

9. Lewis LA, Dyer DW. Identification of an iron-regulated outer membrane protein of Neisseria meningitidis involved in the utilization of hemoglobin complexed to haptoglobin. J Bacteriol 1995; 177: 1299-1306.

10. Thompson SA, Sparling PF. The RTX cytotoxin-related FrpA protein of Neisseria meningitidis is escreted extracellularly by meningococci and by Hly $\mathrm{BD}^{+}$Escherichia coli. Infect Immun 1993; 61: 2906-2911.

11. Thompson SA, Wang LL, West A, Sparling PF. Neisseria meningitidis produces iron-regulated proteins related to the RTX family of exoproteins. $J$ Bacteriol 1993; 175: 811-818.

12. Ala'Aldeen DAA, Wall RA, Borriello SP. Immunogenicity and cross-reactivity of the $70-\mathrm{Kda}$ iron-regulated protein of Neisseria meningitidis in man and animals. $J$ Med Microbiol 1990; 32: 275-281.

13. Ala'Aldeen DAA, Davies HA, Borriello SP. Vaccine potential of meningococcal FrpB: studies on surface exposure and functional attributes of common epitopes. Vaccine 1994; 12: 535-541.

14. Schryvers AB, Gonzalez GC. Comparison of the abilities of different protein sources of iron to enhance Neisseria meningitidis infection in mice. Infect Immun 1989; 57: 24252429.

15. Ala'Aldeen DAA, Powell NBL, Wall RA, Borriello SP. Localization of the meningococcal receptors for human transferrin. Infect Immun 1993; 61: 751-759.

16. Tsai J, Dyer DW, Sparling PF. Loss of transferrin receptor activity in Neisseria meningitidis correlates with inability to use transferrin as an iron source. Infect Immun 1988; 56: 3132-3138.

17. Lissolo L, Maitre-Wilmotte C, Dumas P, Mignon M, Danve B, Quentin-Millet M-J. Evaluation of transferrin-binding protein 2 within the transferrin-binding complex as a potential antigen for future meningococcal vaccines. Infect Immun 1995; 63: 884-890.

18. Rokbi B, Mazarin V, Maitre-Wilmotte G, Quentin-Millet MJ. Identification of two major families of transferrin receptors among Neisseria meningitidis strains based on antigenic and genomic features. FEMS Microbiol Lett 1993; 110: 51-57.

19. Ala'Aldeen DA, Davies HA, Wall RA, Borriello SP. The 70 kilodalton iron regulated protein of Neisseria meningitidis is not the human transferrin receptor. FEMS Microbiol Lett 1990; 69: $37-42$.

20. Wilton J, Ala'Aldeen D, Palmer HM, Borriello SP. Cloning and partial sequence of transferrin-binding protein 2 of Neisseria meningitidis using a novel method: twin N-terminal PCR. FEMS Microbiol Lett 1993; 107: 59-66.

21. Legrain $\mathrm{M}$, Mazarin V, Irwin SW et al. Cloning and characterization of Neisseria meningitidis genes encoding the transferrin-binding proteins $\mathrm{Tbp} 1$ and Tbp 2. Gene 1993; 130: 73-80.

22. Palmer HM; Powell NBL, Ala'Aldeen DA, Wilton J, Borriello SP. Neisseria meningitidis transferrin-binding protein 1 expressed in Escherichia coli is surface exposed and binds human transferrin. FEMS Microbiol Lett 1993; 110: 139-146.

23. Quentin-Millet M-J, Lissolo L, Legrain M et al. Transferrinbinding proteins of Neisseria meningitidis. In: Evans JS, Susan EY, Martin CJM, Feavers IM (eds) Neisseria 94. Potters Bar, UK, NIBSC. 1994: 137.

24. Ala'Aldeen DAA, Borriello SP. The meningococcal transferrinbinding proteins 1 and 2 are both surface exposed and generate bactericidal antibodies capable of killing homologous and heterologous strains. Vaccine (in press).

25. Alcantara J, Yu RH, Schryvers AB. The region of human transferrin involved in binding to bacterial transferrin receptors is localized in the $C$-lobe. Mol Microbiol 1993; 8: 1135-1143.

26. Kabani A, Button L, Schryvers AB. Identification of the 
domains of human transferrin involved in binding to transferrin receptors of human pathogens by the use of chimeric recombinant transferrin expressed in a baculovirun expression system. In: Evans JS, Susan EY, Martin CJM, Feavers IM (eds) Neisseria 94. Potters Bar, UK, NIBSC. 1994: 159-160.

27. Gorringe AR, Irons LI, Aisen $P$, Zak $O$, Robinson $A$. Purification of Neisseria meningitidis transferrin binding proteins and characterisation by epitope mapping and iron release studies. In: Evans JS, Susan EY, Martin CJM, Feavers IM (eds) Neisseria 94. Potters Bar, UK, NIBSC. 1994: 140142.

28. Irwin SW, Averil N, Cheng CY, Schryvers AB. Preparation and analysis of isogenic mutants in the transferrin receptor protein genes, tbpA and tbpB, from Neisseria meningitidis. Mol Microbiol 1993; 8: 1125-1133.

29. Powell NBL, Ala'Aldeen DAA, Schryvers AB, Borriello SP. Electron microscopic localisation of iron-regulated proteins in $N$. meningitidis with particular reference to the lactoferrin and transferrin receptors. In: Evans JS, Susan EY, Martin CJM, Feavers IM (eds) Neisseria 94. Potters Bar, UK, NIBSC. 1994: 150.

30. Ferreirós CM, Ferron L, Criado MT. In vivo human immune response to transferrin-binding protein 2 and other ironregulated proteins of Neisseria meningitidis. FEMS Immunol Med Microbiol 1994; 8: 63-68.

31. Griffiths E, Stevenson P, Byfield $P$ et al. Antigenic relationships of transferrin-binding proteins from Neisseria meningitidis, $N$. gonorrhoeae and Haemophilus influenzae: cross-reactivity of antibodies to $\mathrm{NH}_{2}$-terminal peptides. FEMS Microbiol Lett 1993; 109: 85-92.

32. Cornelissen $\mathrm{CN}$, Sparling PF. Iron-piracy: acquisition of transferrin-bound iron by bacterial pathogens. Mol Microbiol 1994; 14: 843-850.

33. Ala'Aldeen DAA, Davies HA, Wall RA, Borriello SP. Interaction between iron regulated outer membrane proteins of
Neisseria meningitidis and human transferrin binding activity. In: Achtman M, Kohl P, Marchal C, Morelli G, Seiler A, Thiesen B (eds) Neisseria 1990. Berlin, Walter de Gruyter. 1991: 415-418.

34. Stevenson $P$, Williams $P$, Griffiths E. Common antigenic domains in transferrin-binding protein 2 of Neisseria meningitidis, Neisseria gonorrhoeae, and Haemophilus influenzae type b. Infect Immun 1992; 60: 2391-2396.

35. Gerlach GF, Klashinsky S, Anderson C, Potter AA, Willson PJ. Characterization of two genes encoding distinct transferrinbinding proteins in different Actinobacillus pleuropneumoniae isolates. Infect Immun 1992; 60: 3253-3261.

36. Frasch CE. Immunisation against Neisseria meningitidis. In: Easmon CSF, Jeljaszewics J (eds) Medical microbiology. London, Academic Press. 1983: 115-144.

37. Danve $\mathrm{B}$, Lissolo $\mathrm{L}$, Mignon $\mathrm{M}$ et al. Transferrin-binding proteins isolated from Neisseria meningitidis elicit protective and bactericidal antibodies in laboratory animals. Vaccine 1993; 11: 1214-1220.

38. Maitre-Wilmotte $C$, Fourrichon L, Legrain $M$ et al. Mapping monoclonal antibodies directed against meningococcal transferrin-binding protein 2. In: Evans JS, Susan EY, Martin CJM, Feavers IM (eds) Neisseria 94. Potters Bar, UK, NIBSC. 1994: 169.

39. Bishop K, Ala'Aldeen DAA, Powell NBL, Gorringe AR, Borriello SP. Detection of linear and conformational epitopes on the TBP1 component of the meningococcal transferrin receptors, using monoclonal antibodies. J Med Microbiol 1995; 42: 148.

40. Ala'Aldeen DAA, Powell NBL, Borriello SP. Role of antibodies raised during infection in blocking meningococcal transferrin receptors. In: Conde GCJ, Morse S, Rice P, Sparling $\mathrm{F}$, Calderon $\mathrm{E}$ (eds) Pathobiology and immunobiology of Neisseriaceae. Cuernavaca, Mexico, Publications office of the Instituto Nacional de Salud Publica. 1994: 359-362. 